\title{
VIVENCIAS MASCULINAS ANTE EL ALEJAMIENTO FORZADO DE LOS HIJOS
}

\section{MALE LIFE EXPERIENCES FACING FORCED SEPARATION FROM THEIR CHILDREN}

\author{
Pamela González \\ Universidad del BíoBío, Chile \\ Angélica Muñoz \\ Universidad del BíoBío, Chile \\ Nelson Zicavo \\ Universidad del BíoBío, Chile
}

\begin{abstract}
Resumen: Este artículo aborda la comprensión de las vivencias de padres comprometidos con su paternidad acerca del distanciamiento físico y emocional de sus hijos, tras un proceso de separación de pareja. Es un trabajo de relevancia social, pues culturalmente la madre es quien se vincula afectiva y físicamente con los hijos, mientras el padre suele ser considerado el proveedor material de la familia, debiendo alejarse de su prole -por el mandato social- ante la inminente separación de pareja. Al abordar este estudio (desde la fenomenología social) con cinco hombres de la Octava Región, Chile, pudimos concluir que para ellos, dicho distanciamiento, se ha constituido en vivencias estables y dolorosas. Manifestaron el deseo de ser padres y afirmaron poseer cualidades prácticas para cuidar a los hijos a pesar de los obstáculos socio-jurídicos que limitan la continuidad de su paternidad y de sus esfuerzos por mantenerse cerca; esto trae a menudo consecuencias en su vida personal y social, abordadas en este trabajo.
\end{abstract}

Palabras Clave: paternidad, separación de pareja, crianza compartida, vivencias, vínculo.

\begin{abstract}
This paper focuses on the understanding of the experiences of fathers committed to their paternity facing the physical and emotional distance from their children after a partner separation process. From a cultural perspective, it is assumed that the mother is the one who establishes a strong affective and physical bond with the children, while the father is often considered the material provider for the family and is expected to get away from his offspring once the couple disintegrates. The analysis of the cases of five men from the Eighth Region (Chile), from a social phenomenology perspective, shows that for these men the distancing has become a stable painful experience. These men also expressed the desire to be fathers and reported to possess the required qualities to care for their children despite the socio-legal obstacles that limit the continuity of their paternity and their efforts to stay close. This often brings consequences in their personal and social life, which are addressed in this study.
\end{abstract}

Keywords: Paternity, pair separation, shared parenting, experiences, link

Esta investigación se encuentra inserta dentro del proyecto de Investigación interna de la Universidad del BioBio, código DIUBB 1252243/R.

\section{Introducción}

El presente artículo pretende aportar a la comprensión de un fenómeno poco estudiado en Chile; esto es, las vivencias de padres acerca del distanciamiento de sus hijos luego de un proceso de separación de pareja. Este fenómeno se invisibiliza a nivel nacional e internacional, puesto que no se considera a los padres como personas vinculadas efectivamente con sus hijos (Ramírez, 2011). Son concebidos como proveedores materiales de recursos, desvinculados afectivamente, según roles sociales asignados (Ramírez, 2011).
Según estudios de parentalidad, se ha establecido que la figura parental masculina puede ser igual de sensible y tener la misma capacidad de respuesta ante las necesidades y demandas de un hijo que la figura materna (Vallejo, Sánchez-Barraco y SánchezBarraco, 2004; Ferrari, 1999; Zicavo, 2006). Se determinó que los padres que están cerca de sus bebés, ejercen en ellos una influencia cognitiva positiva y significativa (Vallejo et al., 2004). Lo que refuerza la idea de investigar en esta área poco visible socialmente. 


\section{Planteamiento del problema de investigación y justificación}

En Chile, existe una elevada tasa de divorcios, nulidades y separaciones, alcanzando el año 2011 a 47.222 divorcios y 114 nulidades (Servicio de Registro Civil e Identificación, 2012), sin considerar las separaciones en parejas vinculadas por unión de hecho (Viteri, 2011). Así también, la cantidad alarmante de hijos separados de uno de sus padres alcanzó los 95.469 el mismo año. Estos datos permiten reflexionar acerca de la alta cantidad de familias que ven alterado su funcionamiento debido a la disolución conyugal (sea unión de hecho o de derecho), afectando a cada uno de sus integrantes.

Tras las separaciones de pareja, los hijos también se ven afectados, pues en la mayoría de los casos se ven distanciados de uno de sus progenitores. Ferrari (2011) indica que para un niño, el padre y la madre son el mundo, y son todo lo que tiene: si es abandonado por un progenitor, el niño se siente angustiado, más vulnerable que antes, con una valoración resentida de sí mismo. Dicho fenómeno sucede debido a que la custodia compartida no existe en Chile, siendo ejercida sólo por uno de los progenitores (Lathrop, 2010). Culturalmente es el hombre quien se ve distanciado de los miembros del grupo familiar (De la Cruz, 2008; Rodríguez, 2009), viéndose altamente afectado, conductual y emocionalmente, presentando diversos efectos negativos, cuando se encuentra realmente vinculado y preocupado por éstos (Zicavo, 2006; De la Cruz, 2008).

Zicavo y Millahueique (2001) recomiendan profundizar en las investigaciones relacionadas con paternidad, equidad, desigualdad y responsabilidades compartidas, desde diferentes abordajes y perspectivas a dilucidar desde lo masculino, para conocer más sobre estos tópicos. Existen escasos estudios a nivel nacional respecto a las consecuencias psicológicas para el miembro separado de sus hijos (De la Cruz, 2008; Ramírez, 2011), a la interrupción de la paternidad construida desde el compromiso y a la pérdida de espacios gratificantes con la descendencia (Zicavo, 2006).

A nivel social, resulta importante entender una realidad desconocida por causa de la naturalización, puesto que es común pensar que sólo las madres se preocupan y vinculan efectivamente con sus hijos (De la Cruz, 2008), considerando al padre sólo como un proveedor de recursos (Ramírez, 2011). Pero existen padres que están distanciados de sus hijos y pretenden cambiar esta condición y defender el ejercicio de sus derechos (De la Cruz, 2008).

Lo expuesto tiene relevancia metodológica, pues los temas relacionados con la Padrectomía, el Síndrome de Alienación Parental (SAP) y el Síndrome de Padre Devastado (SPD), (asociados con las vivencias acerca del distanciamiento de los padres con sus hijos), se han abordado mediante la combinación de hermenéutica, dialéctica y fenomenología. Se creyó conveniente entonces, un acercamiento (exclusivamente) fenomenológico social a esta temática, buscando conocer el significado que los padres le dan a este distanciamiento.

\section{Objetivos}

\section{Objetivo General:}

Comprender las vivencias de los padres comprometidos con su paternidad acerca del distanciamiento tanto físico como emocional de sus hijos, luego de un proceso de separación de pareja.

\section{Objetivos Específicos:}

1. Comprender el significado que narran los padres comprometidos con su paternidad a la situación de distanciamiento físico-relacional con sus hijos, luego de un proceso de separación de pareja.

2. Comprender los sentimientos de vinculación a partir de las experiencias de vida que generan los padres comprometidos con su paternidad acerca del distanciamiento de sus hijos, luego de un proceso de separación de pareja.

3. Describir las creencias de los padres comprometidos con su paternidad, y que han vivido un proceso de separación de pareja, acerca de la manera en que sus características personales han determinado la forma de vinculación con su hijo.

4. Conocer las experiencias emocionales de distanciamiento que narran los padres comprometidos con su paternidad y que se encuentran separados de sus hijos a esta situación, luego de un proceso de separación de pareja. 


\section{Marco referencial}

Para poder abordar progresivamente el marco referencial, pasamos a delinear la comprensión sobre elementos conceptuales básicos que guiaron nuestro trabajo e implicaron acuerdos relevantes que contribuyen a la comprensión de los casos abordados y las conclusiones posteriores.

Familia: La familia es el ambiente ideal para el desarrollo de los niños ${ }^{1}$ (Ferrari, 2011), promueve el desarrollo social y afectivo de los mismos (Vallejo et al., 2004). Por lo tanto, una familia es un grupo de personas que están unidas emocionalmente como también podrían estarlo por lazos sanguíneos, las cuales han convivido un tiempo suficiente como para haber desarrollado patrones de interacción e historias que justifiquen dichos patrones. Estas personas se construyen entre sí a través de sus interacciones diarias (Minuchin, Lee y Simon, 1998). Sus integrantes comparten un espacio social determinado por relaciones de parentesco, pater-maternidad y conyugalidad (Valdés y Valdés, 2005). Es por ello, que entrega a padres, madres e hijos el derecho de pertenecer a dicho grupo familiar, a permanecer vinculado con este grupo y ejercer la plenitud de sus derechos y deberes independientemente de si la relación conyugal se mantiene o no (Zicavo, 2010).

Separación de Pareja: Se pueden distinguir dos tipos de separaciones: separación de hecho y separación judicial (Véliz, 2004). Así como dos categorías de divorcio: por culpa y por cese irremediable de convivencia (Véliz, 2004).

Cuando existen hijos en la pareja, Zicavo (2010) plantea 4 categorías de divorcio: conyugal, psíquico, emocional y parental. Por lo tanto, el divorcio o la separación ponen fin al matrimonio o unión conyugal, pero no afecta ${ }^{2}$ la filiación ni los derechos y obligaciones que emanan de ésta (Véliz, 2004).

Paternidad: Zicavo (2006) plantea que se debe desvincular la figura del padre con la idea de progenitor. El autor plantea la siguiente definición:

Debemos entender por padre a aquella figura masculina que en su constante intercambio con el niño (en espacio y tiempo adecuados) elige construir junto a su hijo lazos afectivos

1. Es preciso definir en estas líneas que cuando hablemos de niños o hijos nos estaremos refiriendo a masculino y femenino, estableciendo dicha convención con el afán de facilitar la lectura, pero declarando que promovemos el ser inclusivos en lo que a género se refiere.

2. En realidad no debería afectar duraderos en ambas direcciones (padrehijo, hijo-padre) y que es escogido y reconocido por el menor como la figura parental significativa en base al apego emocional desarrollado a través de la convivencia (Zicavo, 2006, p. 66).

En esta investigación se definió paternidad bajo los planteamientos de Ramírez (2011) y Zicavo (2006), como la relación psicofísica estable entre un papá, progenitor o no, y sus hijos. Lo que implica un contacto físico habitual, disponibilidad afectiva recíproca, cooperación en el proceso de custodia y la garantía del pleno desarrollo de las potencialidades de los hijos.

Crianza Compartida: La custodia compartida es una figura que no existe jurídicamente en Chile (Lathrop, 2010). Aun así, esta materia está regulada por el artículo 225 del Código Civil chileno, en donde se indica en sus incisos primero y tercero que, en caso de separación, la madre tendrá el cuidado personal de los hijos pero, en caso de maltrato, descuido u otra causa calificada, el juez puede entregar el cuidado personal al padre, velando así por el interés superior del niño (Lathrop, 2010). Ferrari (2011) refiere que la custodia debe ser concedida a quien garantice mejor la continuidad del vínculo paterno y materno. La crianza o custodia compartida, es la mejor opción para los niños luego de la separación de sus padres, pues así se garantiza que ambos sigan cumpliendo con sus funciones parentales sin atrofiar los vínculos, ya sea por carencia o por exceso (Ferrari, 2011; Vallejo et al., 2004).

Competencias Parentales: Barudy (2005) define las competencias parentales como: "aquellas capacidades prácticas que tienen los padres y/o cuidadores para cuidar, proteger y educar a sus hijos asegurándoles un desarrollo suficientemente sano" (p. 77). Implica satisfacer las necesidades de los niños, tales como: necesidades fisiológicas, cognitivas, sociales, de valores y de protección.

Masculinidad Tradicional: Olavarría y Parrini (2000) conciben el concepto de masculinidad tradicional a partir de ciertas características como lo son el dominio, la autonomía, la fuerza y la potencia; se trata de personas importantes, racionales, con emociones controladas, heterosexuales y proveedores en su familia. Producto de estas conceptualizaciones, no se considera al hombre como un ser apto para ejercer la paternidad afectiva (Elsner, Montero, Reyes y Zegers, 2000; Ramírez, 2011). 
Vínculo: Para Pichon-Rivière (1985), el vínculo corresponde al inter-juego que se ha establecido entre el sujeto y los objetos, siendo una forma de conexión o relación particular para cada caso y momento (Pichon-Rivière, 1985). Para este artículo, se entenderá por vínculo aquella capacidad de apego que tienen los padres con sus hijos y que implica el uso de recursos afectivos, cognitivos y conductuales. En donde se establece una forma de relacionarse particular entre cada padre e hijo (PichonRivière, 1985; Barudy, 2005).

Vivencia: La vivencia es una categoría que comprende la unión de lo afectivo y lo cognitivo, allí se entrelazan necesidades, aspiraciones, deseos y propósitos de una persona (González, 2002). La definimos entonces como un conjunto de acontecimientos instaurados en el continuo de la existencia, siendo inmediatamente entendidos e integrados en la conciencia de cada persona, a través de su propia subjetividad (Doron y Parrot, 2004). Conforme a lo señalado, para efectos de esta investigación y artículo, la vivencia de distanciamiento fue entendida como aquellos acontecimientos respecto a la disolución de los lazos parento-filiales entendidos e integrados por la conciencia subjetiva (Doron y Parrot, 2004; Ramírez, 2011; González, 2002).

Significación: Se consideró como significación de distanciamiento físico-relacional aquellas expresiones de vivencias emocionales referentes a la disolución de los lazos parentofiliales en el ámbito de la interacción recíproca de las partes (Husserl, 1967; Dorsch, Begius y Ries, 2005; Ramírez, 2011).

Sentimientos: Entendemos por sentimientos de vinculación a partir de experiencias previas, aquellos estados afectivos duraderos asociados a la relación recíproca, afectuosa y fuerte, que tenga un padre con su hijo desde las prácticas de vida que haya tenido este padre en su pasado (Heller, 1987; Lersch, 1971; Dorsch et al., 2005).

Creencia: Siguiendo los planteamientos de Zuluaga (2004), Dorsch et al. (2005), se entendió por creencias de vinculación a partir de características personales aquellas frases internalizadas, diálogos privados y lenguaje interior acerca de la relación recíproca, afectuosa y fuerte con su hijo determinadas por las particularidades del padre en el ámbito emocional, cognitivo, cultural, entre otras.

Experiencia: Se concibió por experiencias emocionales de distanciamiento aquel conoci- miento adquirido a partir de percepciones y vivencias propias de carácter histórico, acerca de estados afectivos asociados a la disolución de los lazos parento-filiales (Dorsch et al., 2005; Leclerc-Olive, 2009; Lersch, 1971; Ramírez, 2011).

\section{Antecedentes empíricos}

Durante el año 2011, a nivel país, se registraron 66.132 matrimonios, 47.222 divorcios, 114 nulidades (Servicio de Registro Civil e Identificación, 2012), 50.247 inicios de demandas por separación legal y 100.494 casos de cónyuges que han iniciado el proceso de demanda en los juzgados de familia (Viteri, 2011). Además, desde el año 2005 hasta el año 2010, la cantidad de hijos separados de sus padres alcanzó a 1.024 .320 niños y 375.000 parejas con vínculo de hecho disolvieron sus uniones (Viteri como se citó en Lagos, 2011).

Estudios realizados por el Ministerio de Planificación a través de la Encuesta de Caracterización Socio Económica Nacional (CASEN), evidencian un aumento de la jefatura masculina en familias monoparentales en Chile (Ministerio de planificación, 2009): en el año 1990, el 22,2\% de las familias existentes en el país correspondía a familias monoparentales, equivalente a 841.880 personas (Ministerio de Planificación, 2006), de las cuales un 4,5\% correspondía a jefatura familiar masculina (Ministerio de Planificación, 2009). En tanto, al año 2006, de 73.720 hogares encuestados un $25,6 \%$ de las familias eran monoparentales (Ministerio de Planificación, 2006), de las cuales un 6,1\% tenía una jefatura familiar masculina (Ministerio de Planificación, 2009). Mientras, al año 2009, de un total de 71.460 hogares encuestados, un $27,6 \%$ correspondió a familias monoparentales, de las cuales un $7,2 \%$ corresponde a familias con jefatura masculina (Ministerio de Planificación, 2009).

Bajo estas cifras nace la preocupación de parlamentarios por regular las implicancias de las separaciones de pareja en la totalidad del grupo familiar. Es así como el senador Alejandro Navarro, en el mes de abril del año 2012, detalló un nuevo proyecto de ley que busca regular el 'Síndrome de Alienación Parental' (SAP), mencionando que en Chile la ley propicia el mantenimiento de este proceso de maltrato infantil, con la extendida duración de los juicios (Papá Presente, 2012). Enfatiza que se busca poner fin a la transmisión de rencor de uno de los 
padres hacia su hijo, en contra de la ex-pareja con la que mantiene un conflicto. Por ello, con esta ley se pretende que el cuidado de los hijos sea compartido por ambos padres.

También es destacable señalar que existen organizaciones tanto en Chile como a nivel internacional, que promueven y exigen una paternidad responsable y comprometida con los hijos, a saber: Amor de Papá, Padres por Siempre, AMPFS, También con Papá, APFS, entre otras (Amor de Papá, 2011; Padres por Siempre, 2011; Asociación Mexicana de Padres de Familia Separados, 2012; Corporación de Padres Separados de Chile; 2012).

\section{Material y métodos}

\section{Metodología, diseño y método}

El estudio realizado posee un diseño metodológico de carácter cualitativo, desde un paradigma fenomenológico social, en donde se pretendió comprender las vivencias de padres comprometidos con su paternidad, desde el enfoque biográfico, específicamente a través de la técnica de relato de vida.

La investigación cualitativa posee como objetivo conocer y reconstruir significados captando la información de forma flexible a través de un procedimiento inductivo, lo que se adecúa a esta investigación ya que no se busca la generalización de resultados ni la representación de una población mayor a aquella con la cual se trabajó. Se privilegia entonces, que el auténtico significado de los actos sea conocido por sus protagonistas primando así los aspectos subjetivos a través de las vivencias masculinas asociadas al proceso impuesto de distanciamiento parental de sus hijos (Ruiz, 2007).

\section{Técnicas de recolección}

Se definieron dos técnicas de recolección de información, ambas cualitativas: la entrevista individual en profundidad y la entrevista semiestructurada.

\section{Población}

Se trabajó con cinco personas masculinas, realzando el interés en la profundidad del relato por sobre la extensión de la población, y se utilizó una selección empírica (Ruiz, 2007): padres, sin importar rango etario o si cuentan con pareja actual y nuevos hijos, vinculados efectivamente con sus hijos, donde la separación de pareja no se haya producido por vulneración de derechos en el grupo familiar.

Se utilizó un proceso de muestra en cadena (Hernández, Fernández y Baptista, 2004) y se aplicó un cuestionario que garantizó el trabajo con padres vinculados efectivamente con sus hijos. Se trabajó con tres miembros de la Asociación "Amor de Papá" de la ciudad de Chillán, de los cuales uno de ellos reside en la ciudad de Concepción. Además de dos padres residentes en Chillán, no pertenecientes a ninguna asociación.

\section{Análisis de los resultados}

Se usó un análisis temático, en el cual se enumeraron y explicaron temas relacionados con el campo semántico según el objetivo (Serbia, 2007). Se generó el análisis categorial a través de mallas categoriales con categorías a priori y categorías emergentes (Rodríguez, Gil, y García, 1999). Como señala Flick (2004), en un grupo de personas se encuentran distintas versiones sobre una misma vivencia, por lo que se debió comparar el material empírico con la teoría, dado que el objetivo fue conocer cómo los sujetos investigados conceptualizan su mundo (Rodríguez et al.,1999).

\section{Criterios de calidad}

Consistencia Interna: se triangularon los marcos de investigación (Ruiz, 2007; Rodríguez, Lorenzo y Herrera, 2005), se construyeron instrumentos específicos (Rodríguez et al.,1999) y se sometió la investigación a comentarios de pares (Rodríguez et al., 2005). Se utilizó la Validez Transaccional, velando por el relato contextualizado de las personas (Rodríguez et al., 2005; Taylor y Bogdan, 1987). También se utilizó la Confirmabilidad, usando referencias APA, citaciones y vaciado de datos parcial, permitiendo posterior contraste de información (Rodríguez et al., 2005). Además, se usó grabadora, realizando posteriormente una transcripción fiel de las entrevistas (Castillo \& Vásquez, 2003).

\section{Aspectos éticos}

Al iniciar el trabajo de campo, los entrevistados fueron informados sobre objetivos, métodos, 
usos posibles de la información recogida en las entrevistas y riesgos implicados, garantizándoles anonimato y confidencialidad de su información (Comisión Nacional de Investigación Científica y Tecnológica [CONICYT], 2008; França-Tarragó, 1996). La participación fue voluntaria y se informó bajo qué condiciones el contrato sería anulado. Se utilizó la palabra "entrevistado" para resguardar la privacidad y, en caso de necesitar algún tipo de acompañamiento psicológico posterior, éste sería proporcionado por el "Centro Psicosocial José Luis Ysern de Arce"3 al cual podríamos derivarlos en el momento en que se evaluara fuera imprescindible, situación que no aconteció.

Cada participante firmó un consentimiento informado, conservando una copia de éste (Franca-Tarragó, 1996) y durante el desarrollo de la investigación, las técnicas se usaron de manera graduada, tratando de evitar descompensaciones (Franca-Tarragó, 1996).

\section{Presentación y análisis de los resultados}

1. Objetivo Específico primero: el concepto clave fue "significación de distanciamiento físico-relacional". Se trabajaron las siguientes sub-categorías:

a. Paternidad. Se indagó en cómo los cinco entrevistados definen el concepto de paternidad y cómo eran las relaciones que mantenían con sus hijos. Ante lo cual los sujetos estudiados respondieron que procuran estar con ellos la mayor cantidad de tiempo posible, y que buscaron estar presentes durante todo el proceso de vida del niño, desde la gestación. También, procuran acompañarlos durante el juego, incentivándolos a desarrollar potencialidades y talentos, fortaleciendo la relación.

b. Compromiso Parental. Los cinco hombres estudiados relataron necesitar asumirse y convertirse en padres, afirmando contar con capacidades prácticas y con cualidades que les permiten cuidar, proteger y educar a los hijos, las cuales pudieron desplegar mientras se mantuvieron en contacto con ellos. Exhibieron mediante sus narraciones, el ejercicio de acciones que evidencian compromiso parental acorde con los planteamientos de Barudy (2005), tales como: mantener preocupación por sus hijos, estar al tanto de lo que sucede con el niño y de cómo

3. "Centro Psicosocial José Luis Ysern de Arce", es un centro anexo a la Escuela de Psicología de la Universidad del BíoBío, Chillán, Chile, de trabajo comunitario, social, y clínico dirigido a la población. se está desarrollando, así como procurar aportar en el proceso de construcción identitaria del mismo para que se desarrolle adecuadamente.

c. Padre. Al momento de preguntarle a los cinco entrevistados acerca de cómo se dan cuenta de que sus hijos los reconocen como una figura significativa, y desean que sean sus padres, los sujetos hicieron referencia a frases que los hijos le han mencionado en ocasiones y a la vez, recordaron expresiones donde los niños demostraban que los percibían como una figura que brinda apoyo; algunas acciones y palabras que expresaban el deseo de su presencia, y permanecer junto a éste por tiempos más prolongados que un par de horas. Lo anterior, podría explicarse por los lazos afectivos establecidos con sus hijos desde temprana edad.

d. Significación. Los cinco padres, fueron consultados por la significación que le otorgan a la disolución de los lazos parento-filiales y al hecho de encontrarse distanciados de sus niños no pudiendo llevar a cabo las actividades que realizaban antes en conjunto (o el hecho de verlos con menor frecuencia); ante esto expresan y relatan literalmente que sus vivencias son de intenso dolor difícil de describir para ellos. Se refirieron a la disolución de estos lazos como una de las peores situaciones que han vivido, como un hecho difícil de superar, una pérdida irreparable semejante a la muerte, ante la cual se sienten atados, impedidos y sin oportunidad de revertir la situación pues han perdido lo más importante en su vida, evidenciando cognitiva y emocionalmente un compromiso vivencial real con su paternidad.

e. Desvinculación Parento-Filial. En relación a cómo ha sido la vivencia del proceso de alejamiento de sus hijos para los cinco padres entrevistados, ellos aseveran que han pasado por mucho sufrimiento. Relatan que deben velar desde lejos por los cuidados y la seguridad de éstos, y manifiestan que piensan recurrentemente en cómo se encuentra el niño, expresando que dicha situación les frustra, pues no existe la posibilidad de supervisar de manera cercana las actividades que éste realiza y tampoco pueden actuar en el momento oportuno prestando auxilio. Dicen sentir que con su ausencia (impuesta) dañan al hijo, y que la distancia atenta contra los lazos que en algún momento establecieron; no obstante, los padres dicen mantener esperanza en poder revertir la situación en un futuro, ojalá no lejano. 
f. Distanciamiento Físico-Relacional. Los cinco varones entrevistados afirman que el no poder mantener un contacto físico habitual con los hijos, producto de la dificultad de mantener la convivencia en común con la pareja, les ha imposibilitado mantener una adecuada funcionalidad parental. Así también, declaran que la distancia física facilita la aparición de obstáculos que dificultan la manifestación de una relación afectiva y emocional comprometida, sin importar el esfuerzo que inviertan por mantenerse cerca de sus hijos y acompañarlos en el proceso de crecimiento. Expresan que si no han podido ejercer su paternidad, ha sido por razones externas a ellos, considerando que han estado obligados a la distancia impuesta, pues ésta no ha sido elegida. En relación a esto, hacen mención a la relación que mantienen con la madre del niño, la cual no es armoniosa, de acuerdo a sus criterios, lo que dificulta el encuentro con el menor.

2. Objetivo Específico segundo: el concepto clave fue "sentimientos de vinculación a partir de experiencias previas". Las sub-categorías fueron las que se presentan a continuación:

a. Paternidad. Los cinco hombres mencionaron su compromiso de estar activamente presentes en el proceso de custodia de sus hijos a lo largo de toda la vida, a entregarles afecto, protección y cuidados, así como los lineamientos, normas y reglas que necesiten en idéntica proporción, conforme a las experiencias que han vivenciado a nivel familiar junto a sus padres, madres y sobrinos. Cuentan que a su juicio intentan ofrecerles las mejores condiciones de vida, compartiendo todas las experiencias posibles, estando atentos a sus necesidades y requerimientos materiales y/o emocionales, para garantizarles un sano desarrollo, lo que es concordante con los planteamientos de Zicavo (2006).

b. Experiencia de vida. Los entrevistados repiten modelos o formas de interacción y de vinculación que a su juicio fueron idóneos de parte de sus padres, intentando modificar los aspectos que les parecen desajustados o poco posibles de implementar, como lo fue, en algunos casos, la mayor dedicación de tiempo por parte del propio padre al trabajo y la menor asignación temporal al contacto con ellos. Por esto último, han expresado la intención de evitar que sus hijos pasen por dicha experiencia poco gratificante que ellos debieron vivir a lo largo de su vida, evitando generarles una experiencia inadecuada. c. Sentimientos. Los entrevistados para este estudio, al evocar situaciones en donde se encontraron cerca de sus hijos, reportaron sentimientos positivos como lo son la alegría y felicidad, por tratarse de acontecimientos agradables. Por otra parte, al evocar situaciones desagradables en donde se ven o vieron imposibilitados de mantener un contacto frecuente $y$ regular con sus hijos, mencionan sentimientos negativos tales como: tristeza, miedo, impotencia, rabia y frustración. Reportan que estos últimos son sentimientos constantes y bastante desalentadores por la circunstancia actual en que se encuentran respecto a la relación con su prole.

d. Vínculo. Los cinco padres relatan tener una relación mutua cercana y fuerte con sus niños, a tal punto que éstos últimos sufren ante el alejamiento de sus padres. Por otra parte, aluden a eventos en donde ellos y sus hijos disfrutan del contacto mutuo, encontrando los niños seguridad, confianza y protección en sus padres, los cuales se contactaban con base en el amor, con preocupación y entrega constante.

e. Distanciamiento parento-filial. Cuatro padres mencionan que desde la separación total de sus hijos, la relación ha sido nula por impedimentos impuestos arbitraria y unilateralmente. Salvo en el caso de un sujeto que actualmente tiene derecho a visitas. Dicha imposibilidad de ver a sus hijos, de acuerdo a lo referido, es generada principalmente por su ex-pareja, quien dificulta u obstaculiza las visitas y la mantención de un adecuado contacto con sus hijos. Por lo mismo, según sus reportes, existen consecuencias para ellos y para los menores. En el caso de los menores, éstos sufren ante la separación de sus padres. Y en el caso de los padres la situación resulta totalmente abrumadora, incómoda y dolorosa.

3. Objetivo Específico tercero: el concepto clave fue "creencias de vinculación a partir de características personales". Las sub-categorías fueron las siguientes:

a. Paternidad. Los cinco hombres aseveraron que la responsabilidad era una característica que describía la forma de ejercer su paternidad comprometida, estando conscientes de la importancia de cumplir un rol activo en la vida de sus hijos. Asimismo, su relato evidencia preocupación por el desarrollo y la formación del niño, acorde con los planteamientos de nuestro 
marco teórico, eligiendo un estilo de aproximación afectivo (distante del autoritario), propiciando la cercanía con el hijo y favoreciendo la prolongación vincular anterior.

b. Creencias. Al incursionar en los pensamientos asociados al cómo sus características personales han determinado el vínculo con los hijos y la relación recíproca y afectuosa establecida con éstos, la población estudiada hizo mención a los buenos modales que han presentado frente a los niños, los cuales fomentan el respeto mutuo. Asimismo, la capacidad de expresar cariño y emocionarse ha permitido que los niños respondan de igual forma ante el contacto y la cercanía con el padre. Algunos padres también creen que características más lúdicas inherentes a sí mismos lograron dar respuesta a las necesidades de sus hijos y fortalecieron la relación que pudieron establecer mientras conservaron el contacto paternal.

c. Características Personales. Toda la población que participó en esta investigación fue capaz de mencionar ciertos elementos que los caracterizan, los cuales a su parecer, favorecieron el tipo de vínculo que mantuvieron o mantienen con su hijo. En este sentido, ellos aludieron a sus características lúdicas, a la capacidad de expresar emociones y sentimientos, el contar con habilidades para expresarse verbalmente, exhibirse como una persona respetuosa, ser cariñoso, demostrar honestidad y responsabilidad, junto al deseo de mantenerse presente y activo en la vida de sus niños.

d. Vinculación Parento-Filial. Al preguntar acerca del tipo particular de relación establecida entre los padres y sus hijos, éstos señalaron que existía una relación basada en la confianza, en el afecto, sin ejercer un trato autoritario. En relación a ello, un padre señala que desarrolló una relación igualitaria, de pares.

De esa forma, estos hombres relatan que procuraron mantenerse cerca de sus hijos, y cautelar por el cuidado de éstos, protegerlos y dejarles enseñanzas, dentro del tiempo que se les dispuso para ello.

4. Objetivo Específico cuarto: el concepto clave fue "experiencias emocionales de distanciamiento". Las sub-categorías fueron las siguientes:

a. Paternidad. Los cinco entrevistados mencionan que lamentan y sufren al no permanecer cerca de los hijos y ejercer activamente su paternidad, relatando que los dañan tanto a ellos como a los chicos, ya que han perdido etapas valiosas de su desarrollo y crecimiento. No importa que el tiempo sea reducido para estar cerca de los niños, señalan que sin dudas no es lo ideal, pero es mejor eso a tener un contacto nulo con quien más aman. Reportan además que las situaciones vividas junto a sus hijos siempre son gratas y enriquecedoras, por lo que anhelan mantener un contacto regular y duradero en el tiempo.

b. Compromiso parental. Todos los entrevistados para efectos de este estudio, de acuerdo a sus relatos, respetaron (cuando existieron), los acuerdos judiciales que regulaban los derechos de visitas con sus hijos. Enfatizando que entregaban tiempo de calidad a los niños, el cual intentaban que fuera disfrutado y aprovechado al máximo. Al respetar dichos acuerdos, según sus propios relatos, se encuentran comprometidos con el ejercicio efectivo y activo de su paternidad, cuando se les permite, propiciando un adecuado desarrollo en los niños.

Finalmente, hacen referencia a su interés por evitar el daño o sufrimiento en sus hijos, aunque muchas veces no pueden lograrlo en su totalidad, pues se encuentran distanciados de ellos, y según sus propios reportes, dichas situaciones los dañan tanto a ambas partes.

c. Experiencia emocional. Las experiencias relacionadas con la disolución de lazos parento-filiales, de acuerdo a sus relatos, están referidas a sentimientos negativos: rabia, tristeza, frustraciones, incomprensión, angustia, desesperación, entre otras. Así también, dicha incomodidad y emocionalidad negativa afecta todos los aspectos de la vida de los entrevistados, con graves consecuencias en su vida y sus relaciones sociales, según reportan, ya que se sienten invalidados e imposibilitados para ejercer su paternidad, a pesar de los esfuerzos que han realizado y de las capacidades que han demostrado poseer. Refieren además, sentimientos permanentes de culpa por el distanciamiento impuesto con sus hijos.

d. Distanciamiento parento-filial. Los padres hacen referencia a que el distanciamiento con sus hijos fue inesperado. Jamás esperaron el tipo de reacciones que han tenido sus ex-parejas con ellos y con los niños, imposibilitándoles el derecho de ejercer activamente su paternidad, interfiriendo todos los aspectos involucrados en el ejercicio pleno de su derecho a ser padres, afectando no solo a los adultos, sino también a los chicos, como queda evidenciado en las narraciones de los cinco sujetos. 
Así también, los entrevistados reportan que producto de este distanciamiento han perdido valiosos momentos y etapas significativas dentro del desarrollo de sus hijos que nadie debiera desperdiciar (tanto físico, como emocional), calificando la situación de injusta y dolorosa.

5. Categorías Emergentes: Las siguientes categorías se presentaron de manera recurrente en el relato de los entrevistados, resultando ser hallazgos del proceso investigativo, por lo que han sido integradas dentro del proceso de análisis.

a. Errores en las Resoluciones Judiciales. Constantemente los cinco entrevistados reportan fuertes percepciones de injusticia y malas o incorrectas sentencias de parte del sistema judicial de familia, considerando que se han cometido muchos errores e irregularidades. Lo cual está ligado a las normativas y leyes imperantes en Chile, pues la custodia compartida no es una figura jurídica en nuestro país y todas las decisiones judiciales se amparan en el artículo 225 del Código Civil chileno. En dicho artículo se establecen las ventajas en el cuidado y tuición de los hijos que presentan las madres por sobre los padres; resultando esto una discriminación a todas luces o al menos evidenciando un estatus jurídico inferior de los padres basado en características biológicas que agregan un sesgo marcado donde se supone igualdad ante la ley. Por otra parte, esto pudiera ocurrir también por la carencia de capacitación adecuada en temas específicos de parentalidad (Ramírez, 2011), Alienación (Aguilar, 2005) y Padrectomía (Zicavo, 2006) de los magistrados y abogados involucrados en el proceso.

b. Consecuencias en la Vida de los Padres Producto del Distanciamiento Parento-Filial. El distanciamiento abrupto o paulatino de la vida de los hijos producto de la separación de pareja, según el relato de los participantes, revela que conlleva profundas consecuencias en sus vidas. Ya sea en los aspectos personales, como lo son las relaciones de pareja que establecen y el desarrollo personal, o sus relaciones laborales y sociales. Lo que les genera dificultades para continuar con una vida adecuada, equilibrada. La ruptura de pareja condiciona y conduce a la ruptura de la parentalidad masculina con consecuencias dramáticamente inesperadas.

\section{Conclusiones}

Es relevante recordar que debido a las características metodológicas y epistemológicas de la presente investigación, se trabajó con una población acotada de cinco participantes, por lo que los resultados y conclusiones de la misma se realizan en base a sus relatos y vivencias cotejados con la literatura y el conocimiento existente en la temática, no debiendo ser extrapolados a una población mayor.

Es por ello, que respecto a la "comprensión del significado que le entregan los cinco padres comprometidos con su paternidad, al distanciamiento tanto físico como relacional de sus hijos, luego de una separación de pareja", correspondiente al primer objetivo específico de investigación, se observa que para los entrevistados se trata de vivencias abrumadoras, dolorosas, pues narran que la disolución de los lazos parento-filiales ha sido uno de los sucesos más dramáticos que les ha ocurrido. Este distanciamiento ha sido difícil de superar, ya que han procurado estar con sus hijos la mayor cantidad de tiempo posible, sin importar la situación de pareja en la que se encuentren, coincidiendo con lo expuesto por Zicavo y Millahueique (2001). Han expresado que es una pérdida semejante a la muerte, ante la cual se sienten atados, dado que se han esforzado por revertir la situación y no lo han logrado. Han perdido lo más importante en su vida.

Los cinco participantes han manifestado el deseo de convertirse en padres, y han afirmado que cuentan con capacidades prácticas para cuidar, proteger y educar a los hijos. Señalan que los hijos les han demostrado a través de acciones y palabras que los percibían como una figura que brinda apoyo, además de expresarles el deseo de su presencia por más de un par de horas, todo esto en base al apego emocional que desarrollaron mediante la convivencia, lo que corrobora lo expresado por Zicavo (2006). Sin embargo, la distancia física impuesta producto de la separación de pareja, facilita la aparición de obstáculos que atentan contra los lazos que establecieron antes con sus hijos pues, tal como señala Goldberg (2004), este alejamiento enfría el vínculo y acentúa la distancia psicológica, emocional, sin importar los esfuerzos que ellos realicen por mantenerse cerca. Estos padres expresan que si no han podido ejercer su paternidad, ha sido por razones externas (e injustas) a ellos, aludiendo 
a que sería una consecuencia arbitraria de las distancias relacionales con la madre del niño.

Deben preocuparse desde lejos por los cuidados y la seguridad de los menores, sin poder supervisar constantemente las actividades que realizan ni auxiliarles oportunamente, tal como lo afirma Zicavo (2010), pues en nuestra realidad nacional y cultural es el padre quien debe alejarse del grupo familiar que contribuyó a formar, sólo por considerarse socialmente como el proveedor material en la familia. Por ello, se encuentran pensando recurrentemente en cómo se encuentra el niño, lo que los frustra muchas veces, pero mantienen la esperanza de revertir la situación (Zicavo, 2010).

El segundo objetivo específico de la investigación, fue "comprender los sentimientos de vinculación a partir de experiencias de vida que generan padres comprometidos con su paternidad acerca del distanciamiento de sus hijos, luego de un proceso de separación de pareja". Ante esto, se observa que los sentimientos experimentados por los participantes en relación a dicho proceso son desalentadores, pues al evocar situaciones en donde se ven o vieron imposibilitados de mantener un contacto frecuente y regular con sus hijos, señalan experimentar sentimientos negativos tales como: tristeza, miedo, impotencia, rabia y frustraciones. Reportando que son sentimientos constantes, estables y permanentes en el tiempo, concordando con lo planteado por Lersch (1971). Por lo tanto, cuando una vivencia se prolonga en el tiempo, ya sea relacionada con el placer o el displacer, se convierte en un estado de ánimo de displacer permanente, asociado y producto de una vivencia particular (Dorsch et al., 2005), en este caso, las vivencias negativas son producto del distanciamiento sufrido involuntariamente.

Las vivencias dolorosas de estos cinco hombres, concuerdan con las características expuestas por Zicavo (2006) sobre el Síndrome del Padre Devastado (SPD): aquel conjunto de síntomas vividos emocional y conductualmente por un padre, tras la pérdida de un hijo en el proceso de post-divorcio. A pesar de esto, los padres son capaces de generar sentimientos positivos al recordar situaciones alegres y cercanas con sus hijos: sentimientos de alegría y felicidad. Siendo además el amor, respeto y confianza elementos transversales en sus relatos mientras se referían a sus hijos, independiente del recuerdo. Dichos sentimientos están ligados a las experiencias de vida de los participantes, a aquellas vivencias incorporadas en los padres, las cuales actúan desde el pasado en sus vivencias presentes, donde la fantasía, la memoria y la imaginación se encuentran implicadas (Lersch, 1971; Heller, 1994). Según relatan, ellos repiten las formas de interacción y de vinculación que a su juicio fueron idóneos de parte de sus padres, intentando modificar aquellos aspectos que les parecen poco oportunos de implementar con sus hijos. Lo que es consecuente con los planteamientos de Bollnow (2001), quien manifiesta que la experiencia no se adquiere a través de oír la narración de otra persona, sino más bien es el resultado del constante enfrentamiento con los hechos y el hacer en el propio cuerpo, ya que éstas no se transmiten.

Sobre el tercer objetivo, "describir las creencias de los padres comprometidos con su paternidad, y que han vivido un proceso de separación de pareja, acerca de la manera en que sus características personales han determinado la forma de vinculación con su hijo", los entrevistados mencionaron los buenos modales exhibidos ante los niños. Aludieron a sus habilidades para expresarse verbalmente, ser respetuosos, honestos y responsables, junto al deseo de mantenerse presente en la vida de sus niños. Dichos elementos, a su juicio, fomentaron el respeto mutuo, además de creer que su capacidad de expresar cariño y emocionarse ha permitido que los niños respondan de igual forma ante su contacto y cercanía. Algunos padres también creen que sus características más lúdicas inherentes a su individualidad respondieron a las necesidades de sus hijos.

Los cinco hombres mencionaron ciertos elementos que los caracterizan, los cuales a su parecer, favorecieron el tipo de vínculo que mantuvieron o mantienen con sus hijos. Un vínculo basado en la confianza, en el afecto y, en algunos casos, una relación igualitaria, sin la necesidad de ejercer un trato autoritario. Dicho vínculo se ha vivenciado desde antes de la separación de pareja, por lo que suele estar presente en la vida de los entrevistados con naturalidad y la necesidad de ser mantenido en el tiempo.

Es fundamental crear una relación de vinculación afectiva o de apego entre los niños y sus figuras significativas, ya que éstas aportan con sus experiencias y apoyo a enfrentar el mundo (Vallejo et al., 2004). Por lo expuesto, estos hombres han procurado desde antes mantenerse cerca de sus hijos, cuidándolos y educándolos, mientras les fue permitido.

Como plantea Ferrari (2011), la familia debe seguir conteniendo afectivamente, manejando 
afectos, brindando seguridad, a pesar de que la pareja de padres se haya separado, y los padres entrevistados procuran cumplir con esto.

En cuanto a "conocer las experiencias emocionales de distanciamiento que entregan los padres comprometidos con su paternidad y que se encuentran separados de sus hijos, luego de un proceso de separación de pareja", último objetivo específico de la investigación, se concluye que los padres entrevistados vivencian emocionalmente el distanciamiento de sus hijos como un evento muy complejo y difícil, asociado a sentimientos negativos como rabia, tristeza, frustraciones, incomprensión, angustia, desesperación y culpa, que concuerdan con la definición de Síndrome de Padre Devastado de Zicavo (2006). Siendo permanentes en el tiempo los sentimientos de tristeza y rabia. Por lo que este conocimiento adquirido por los participantes de la investigación en relación a sus vivencias y percepciones propias, es un conocimiento que permanecerá a lo largo de toda su vida y será constantemente evaluado (Dorsch et al., 2005). Dichos sentimientos son producto de aquello que fue percibido, sentido o pensado por los padres en relación al mundo que los rodea (Doron y Parrot, 2004), en este caso todo el proceso de distanciamiento emocional y físico de sus hijos y la imposibilidad de ejercer adecuadamente su paternidad, es lo que repercute en todos los ámbitos de la vida de los sujetos, como ellos mismos reportaron, tendrá consecuencias en su vida personal y social.

Son profundas consecuencias, muchas veces permanentes en el tiempo, que traspasan todos los aspectos de las vidas de los sujetos, dificultando su desarrollo personal, relaciones de pareja y rendimiento laboral (Zicavo, 2006).

También, los padres evidencian percepciones de injusticia y malas (e inadecuadas) sentencias de parte del sistema judicial, señalando errores graves en su funcionamiento, debido a que en Chile no existe la custodia compartida como figura legal (Lathrop, 2010). Dichas temáticas se regulan por el artículo 225 del Código Civil chileno, en donde se indica en sus incisos primero y tercero que, en caso de separación, la madre tendrá el cuidado personal de los hijos pero, en caso de maltrato, descuido u otra causa calificada, el juez puede entregar el cuidado personal al padre, velando por el interés superior del niño (Lathrop, 2010). Por ello, los entrevistados perciben poca comprensión por parte de la sociedad, percepción avalada por las constantes dificultades que han enfrentado para regularizar sus relaciones con sus hijos, sean éstas impuestas por la madre de los menores o por la propia justicia.

Por último, queda planteada la necesidad de continuar investigando en la temática, idealmente con una población mayor a la utilizada para este trabajo, resaltando las consecuencias en la vida de los padres producto del distanciamiento parento-filial, para generar conocimientos nuevos y/o complementar y corroborar lo existente. Avalando que los padres comprometidos con sus hijos existen, son una fuerza social creciente y les interesa seguir presente en la vida de éstos. Resulta importante generar investigaciones con poblaciones similares a ésta, que utilicen otro enfoque epistemológico, que permita el trabajo con una población más amplia y representativa desde una perspectiva cuantitativa de la problemática planteada, en otras áreas del país y del continente, emanando conocimientos asociados al distanciamiento parento-filial y sus consecuencias tanto para los padres, como para los menores, temática que no fue trabajada en esta investigación y que sería interesante indagar para reforzar lo aquí planteado. Debemos tener en cuenta que las parejas se divorcian, se separan, pero los padres no deben hacerlo pues la crianza es para toda la vida. Los hijos necesitan a ambos padres no sólo cuando pequeños sino desde y hasta siempre para construir y construirse como individuos independientes y sanos psicológicamente, esa es la responsabilidad social que nos compete a todos.

\section{Referencias}

Aguilar, J. (2005). S.A.P. Hijos manipulados por un cónyuge para odiar al otro. España; Almuzara.

Amor de Papá. (2011). Amor de Papá. Recuperadp de http:// www.amordepapa.org

Asociación Mexicana de Padres de Familia Separados. (2012). Recuperado de http://ampfsmexico.blogspot.com/

Barudy, J. (2005). Los buenos tratos a la infancia: parentalidad, apego y resiliencia. Barcelona: Gedisa.

Bollnow, O. (2001). Introducción a la filosofía del conocimiento. La comprensión previa y la experiencia de lo nuevo. Buenos Aires: Amarrortu editores.

Castillo, E. y Vásquez, M. (2003). El rigor metodológico en la investigación cualitativa. Colombia Médica, 34 (3): 164-167.

Comisión Nacional de Investigación Científica y Tecnológica. (2008). Bioética en investigación en ciencias sociales. Santiago: Gobierno de Chile. 
Corporación de Padres Separados de Chile. (2012). Recuperado de http://www.apfs.cl/index-2.htm

De la Cruz, A. (2008). Divorcio destructivo: cuando uno de los padres aleja activamente al otro de la vida de sus hijos. Revista Diversitas, 4 (1): 149-157.

Doron, R. y Parrot, F. (2004). Diccionario Akal de Psicología. Madrid: AKAL.

Dorsch, F., Begius, R. y Ries, H. (2005). Diccionario de psicología. (7a ed.) Barcelona: Herder.

Elsner, P., Montero M., Reyes C. y Zegers, B. (2000). La familia: una aventura. Santiago: Universidad Católica de Chile.

França-Tarragó, O. (1996). Ética para psicólogos. Introducción a la psicoética. Bilbao: Desclée de Brouwer.

Ferrari, J. (1999). Ser padre en el tercer milenio. Mendoza: Canto Rodado.

Ferrari, J. (2011). Padre amado o deseado: La nueva relación entre padres e hijos. México: Trillas.

Flick, U. (2004). Introducción a la investigación cualitativa. Madrid: Morata.

Goldberg, B. (2004). Tuyos, míos, nuestros: cómo rearmar y disfrutar la familia después del divorcio. Buenos Aires: Lumen.

González, F. (2002). Sujeto y subjetividad: una aproximación histórico-cultural. México D.F.: International Thomson

Heller, A. (1987). Teoría de los sentimientos. Tlahuapán: Distribuciones Fontamara.

Heller, A. (1994). Sociología de la vida cotidiana. Barcelona: Península.

Hernández, R., Fernández, C. y Baptista, P. (2004). Metodología de la investigación. México: Mc Graw-Hill.

Husserl, E. (1967). Investigaciones lógicas I-II. Madrid: Revista de Occidente.

Lagos, P. (2011). El síndrome de alienación parental: una realidad latente. Recuperado de http://www.emol. com/tendenciasymujer/Noticias/2011/03/11/20894/ El-sindrome-de-alienacion-parental-una-realidadlatente.aspx

Lathrop, F. (2010). Custodia compartida, acuerdo de los padres y establecimiento de oficio: un fallo en ausencia de ley. Revista de Derecho, 23 (2): 237-245.

Leclerc-Olive, M. (2009). Temporalidades de la experiencia: las biografías y sus acontecimientos. Iberoforum, 4 (8): 1-39.

Lersch, P. (1971). La estructura de personalidad. Barcelona: Scientia.

Ministerio de Planificación (2006). Encuesta de caracterización socioeconómica nacional. Recuperado de http:// observatorio.ministeriodesarrollosocial.gob.cl/layout/ doc/casen/publicaciones/2006/Resultados_Familia_ Casen_2006.pdf

Ministerio de Planificación (2009). Encuesta de caracterización socioeconómica nacional. Recuperado de http://www. ministeriodesarrollosocial.gob.cl/casen2009/familia. php

Minuchin, S., Lee, W. y Simon, G. (1998). El arte de la terapia familiar. Barcelona: Paidós.
Olavarría, J. y Parrini, R. (2000). Masculinidad/es identidad, sexualidad y familia. Santiago: Flacso.

Padres por Siempre. Corporación padres por igualdad de derechos frente a los hijos (2011). Recuperado de http://www.papasporsiempre.cl/

Papá Presente. (2012). Proponen establecer cuidado compartido de menores y evitar el daño en caso de separación de los padres. Recuperado de http:// www.papapresente.org/informaciones_detalle. asp?Solicitud $=275$

Pichon-Rivière, E. (1985). Teoría del vínculo. Buenos Aires: Nueva Visión.

Ramírez, D. (2011). La desparentalización impuesta al padre, separado o divorciado: secuelas psicosociales. San José: Ediciones Universitarias.

Rodríguez, M. (2009). El cuidado personal de niños y adolescentes en la familia separada: criterios de resolución de conflictos de intereses entre padres e hijos en el nuevo derecho chileno de familia. Revista Chilena de Derecho, 36 (3): 545-586.

Rodríguez, G., Gil, J. y García, E. (1999). Metodología de la investigación cualitativa. Málaga: Aljibe.

Rodríguez, C., Lorenzo, O. y Herrera, L. (2005). Teoría y práctica del análisis de datos cualitativos. Proceso general y criterios de calidad. Revista Internacional de Ciencias Sociales y Humanidades, SOCIOTAM, 15 (2): 133-154.

Ruiz, J. (2007). Metodología de la investigación cualitativa. Bilbao: Universidad de Deusto.

Serbia, J. (2007). Diseño, muestreo y análisis en la investigación cualitativa. Hologramática, 4 (7):123-146.

Servicio de Registro Civil e Identificación. (2012). Estadísticas con enfoque de género. Recuperado de http://www. registrocivil.gob.cl/PortalOI/f_estadisticas_enfoque de_genero.html

Taylor, S. y Bogdan, R. (1987). Introducción a los métodos cualitativos de investigación. Barcelona: Paidós.

Valdés, X. y Valdés, T. (2005). Familia y vida privada, ¿Transformaciones, tensiones, resistencias o nuevos sentidos? Santiago de Chile: Flacso.

Vallejo, R., Sánchez-Barraco, F. y Sánchez-Barraco, P. (2004). Separación o divorcio: trastornos psicológicos en los padres y los hijos. Revista de la asociación española de neuropsiquiatría. (92): 91-110.

Véliz, P. (2004). Divorcio, nulidad y separación: los caminos frente a la ruptura. Santiago: Cerro Manquehue.

Viteri, R. (2011). Estadísticas. Recuperado de www. separadosdechile.cl

Zicavo, N. (2006). ¿Para qué sirve ser padre? Concepción: Ediciones Universidad del Bío-Bío.

Zicavo, N. (2010). Crianza compartida. México: Trillas.

Zicavo, N., \& Millahueique, E. (2001). El nuevo rol paternal afectivo y cercano. Annales, 14: 405-429.

Zuluaga, D. (2004). La terapia cognitiva: una revisión general. Perspectivas en Psicología, 6 (6): 59-66.

Para citar este artículo:

González, P., Muñoz, A. \& Zicavo, N. (2014). Vivencias masculinas ante el alejamiento forzado de los hijos. Ciencias Psicológicas VIII (1): 43 - 54 